\title{
Autophagy inhibition enhances etoposide-induced cell death in human hepatoma $\mathbf{G} 2$ cells
}

\author{
BU-SHAN XIE ${ }^{1,2}$, HONG-CHUAN ZHAO $^{2}$, SHU-KUN YAO ${ }^{2}$, DE-XIANG ZHUO ${ }^{3}$, BO JIN ${ }^{3}$, DUI-CAI LV ${ }^{3}$, \\ CHENG-LIN WU ${ }^{3}$, DAI-LONG MA ${ }^{3}$, CHUN GAO $^{1,2}$, XIAO-MING SHU ${ }^{1,2}$ and ZHENG-LIN AI ${ }^{1,2}$ \\ ${ }^{1}$ Graduate School, Peking Union Medical College and Chinese Academy of Medical Sciences, Beijing 100730; \\ ${ }^{2}$ Department of Gastroenterology, China-Japan Friendship Hospital, Ministry of Health, Beijing 100029; \\ ${ }^{3}$ Department of Biochemistry and Molecular Biology, Health Science Center, \\ Peking University, Beijing 100191, P.R. China
}

Received October 19, 2010; Accepted December 20, 2010

DOI: $10.3892 / \mathrm{ijmm} .2011 .607$

\begin{abstract}
Induction of autophagy usually acts as a survival mechanism of cancer cells in response to chemotherapy. However, the function and molecular mechanism of autophagy in human hepatoma cells under drug treatment is still not clear. To address this issue, we established an experimental model in which HepG2 cells were treated with etoposide, a widely used anticancer agent. We demonstrate the etoposide-induced accumulation of GFP-LC3 dots by fluorescent microscopy, the up-regulation of LC3-II protein expression by Western blotting and the increased number of autophagic vacuoles by electron microscopy, confirming the activation of autophagy by etoposide in HepG 2 cells. Inhibition of autophagy by either 3-methyladenine (3MA) or beclin-1 small interfering RNA enhanced etoposide-induced cell death. Furthermore, activation of p53 and AMPK was detected in etoposide-treated cells and inhibition of AMPK triggered apoptosis through suppression of autophagy. On the other hand, inactivation of p53 promoted cell survival through augmentation of autophagy. Collectively, these findings indicate that etoposide-induced autophagy promotes hepatoma cell adaptation and survival, and that autophagy inhibition improves the chemotherapeutic effect of etoposide. Moreover, AMPK activation is clearly associated with etoposide-induced autophagy. We conclude that manipulation of AMPK may be a promising approach of adjuvant chemotherapy for hepatocellular carcinoma.
\end{abstract}

\section{Introduction}

Hepatocellular carcinoma (HCC) is the most common type of liver cancer and its incidence is rather high in most East Asian

Correspondence to: Dr Shu Kun Yao, Department of Gastroenterology, China-Japan Friendship Hospital, Ministry of Health, Beijing 100029, P.R. China

E-mail: yaosk@zryhyy.com.cn

Key words: autophagy, etoposide, beclin-1, AMP-activated protein kinase, p53 countries (1-3). Early diagnosis and surgery is the optimal way to treat $\mathrm{HCC}$, yet most people at the time of diagnosis have advanced or metastatic symptoms. Adjuvant chemotherapy is an integral component of care for HCC patients with metastatic symptoms. Etoposide, a DNA-damaging agent, is frequently used as adjuvant chemotherapy for a wide variety of human malignant tumors, including HCC (4-6). The primary cytotoxic target for etoposide is the topoisomerase II-DNA cleavage complex, accumulating in drug-treated cells causing DNA strand breaks and accordingly inducing p53-dependent apoptosis $(7,8)$. However, use of etoposide as a single agent for HCC treatment has been associated with a low response rate and with cancer recurrence (9). Furthermore, the results of combination chemotherapy for primary liver cancer from an Eastern Cooperative Oncology Group trial are disappointing (10). Therefore, novel therapeutic methods are required to improve the poor treatment response of HCC.

Increasing evidence reveals that induction of autophagy plays a self-defensive role under chemotherapy and its inhibition enhances antineoplastic agent-induced cell death. Autophagy is a general term for the degradation of cytoplasmic components, such as the turnover of long-lived proteins and organelles in lysosomes (11). Although autophagy was initially identified as a process induced by amino acid starvation promoting cell adaptation (12), the role of autophagy in cancer is not clearly defined. Under stressful conditions, the contribution of autophagy as a survival mechanism in cancer has been well recognized. But, in some situations, autophagy, often as programmed cell death, kills cancer cells.

AMP-activated protein kinase (AMPK) is a multisubunit enzyme that is considered a central metabolic regulator found in all eukaryotes, governing glucose and lipid metabolism in response to nutrient starvation and energy deficiency (13). A broader function of AMPK in cellular homeostasis has been suggested (14). In addition to its role in normal glycolytic and oxidative metabolism, inhibition of the mammalian target of rapamycin (mTOR) by AMPK might be expected to induce autophagy (15). It has generally been accepted that mTOR, a pivotal regulator for autophagy, can be activated by AMPK.

In this study, stimulation of autophagy was detected in etoposide-treated HepG2 cells. Furthermore, suppression of 
autophagy enhanced etoposide-induced cell death. Inhibition of AMPK enhanced cell death through autophagy inhibition. Based on these results, we conclude that etoposide-induced autophagy functions to maintain cancer cell viability, and is closely related to AMPK activity; thus, manipulation of AMPK activity through inhibition of autophagy might be a feasible tumor treatment in future clinical applications.

\section{Materials and methods}

Materials. 3-Methyladenine (3MA), compound C, pifithrin- $\alpha$ (PFT $\alpha$ ), antibody against microtubule-associated protein 1 light chain 3 (LC3) were purchased from Sigma Chemical Co. (St. Louis, MO). Etoposide was purchased from Hengrui Medical Company (JiangSu, China). The antibody against p70s6 (Thr389) and pAMPKa (Thr172) was obtained from Cell Signaling Technology, Inc. (Beverly, MA, USA), whereas antibodies against beclin-1, p53 and $\beta$-actin were from Santa Cruz Biotechnology.

Tumor cells and the MTT (3-(4,5-dimethylthiazol-2-yl)-2, 5-diphenyltetrazolium bromide) assay. HepG2 cells (obtained from ATCC) were routinely cultured in Dulbecco's modified Eagle's medium (Invitrogen, Carlsbad, CA, USA) supplemented with $10 \%$ (v/v) fetal bovine serum (FBS) (Invitrogen), $100 \mathrm{kIU} / 1$ penicillin (Invitrogen), and $100 \mathrm{mg} / \mathrm{l}$ streptomycin (Invitrogen), at $37^{\circ} \mathrm{C}$ in an humidified atmosphere containing $5 \% \mathrm{CO}_{2}$. Flasks were subcultured every 2 days with a split ratio 1:2. Cells were seeded in 96-well flat bottom microtiter plates. After etoposide treatment, $100 \mu 1 \mathrm{MTT}(5 \mathrm{mg} / \mathrm{ml}$; Sigma) per well was added and incubated for $4 \mathrm{~h}$. The crystals were dissolved in $100 \mu \mathrm{l}$ DMSO. The absorbance of the solution was read spectrophotometrically at $570 \mathrm{~nm}$ using a microtiter plate reader (Becton-Dickinson).

Fluorescence microscopy. Cells at approximately $60 \%$ confluency were transfected with GFP-LC3 using Lipofectamine 2000 reagent (Invitrogen), were then subjected to various treatments and observed with a Leica fluorescence microscope (Germany). The percentages of cells with punctate GFP-LC3 structures were obtained from five non-overlapping fields and the statistical data were obtained from three repeated experiments.

Electron microscopy. Cells were harvested by trypsinization, washed with PBS and fixed with ice-cold glutaraldehyde (3\% in $0.1 \mathrm{M}$ cacodylate buffer, $\mathrm{pH} 7.4$ ) for $30 \mathrm{~min}$. The etoposide-treated or untreated cells were post-fixed in $\mathrm{OsO}_{4}$ and embedded in Epon; $0.1 \mathrm{~mm}$ thin sections were stained with uranyl acetate/lead citrate (Fluka) and viewed with a JEM1230 electron microscope (Japan).

Flow cytometry analysis. Cells were trypsinized (keeping all floating cells), and washed with cold PBS and then resuspended in $1 \mathrm{X}$ Binding Buffer at a concentration of $1 \times 10^{6}$ cells/ml. Cells were incubated with Annexin V-fluorescein isothiocyanate (FITC) (BD Pharmingen) for $30 \mathrm{~min}$ at $4^{\circ} \mathrm{C}$ in the dark, then incubated with propidium iodide (PI) (BD Pharmingen) for $2 \mathrm{~min}$ and immediately analyzed by flow cytometry (FACSAria, Becton-Dickinson).
Small interfering RNA (siRNA) transfection. Cells were seeded at $30-50 \%$ confluency per well in 6-well plates overnight and transfected with targeted-gene siRNA or control siRNA duplex (Dharmacon Research, Lafayette, CO), and then cultured with various treatments for further experiments. Successful blockage of the target gene was examined by immunoblotting analysis.

Western blot analysis. Cells were pelleted by centrifugation and lysed in lysis buffer (10 mM HEPES pH 7.4; $0.15 \mathrm{M} \mathrm{NaCl}$, $1 \mathrm{mM}$ EDTA, $1 \mathrm{mM}$ EGTA, 1\% Triton X-100, 0.5\% NP-40, with freshly added PMSF) for $30 \mathrm{~min}$ on ice. Cell lysates were centrifuged at the maximum speed for $15 \mathrm{~min}$ at $4^{\circ} \mathrm{C}$. In addition, proteins were detected by using SDS-PAGE electrophoresis and transferred onto nitrocellulose membranes. After blocking with $5 \%$ non-fat milk, the membranes were incubated with the primary antibodies overnight at $4^{\circ} \mathrm{C}$, followed by the secondary antibodies at room temperature. Protein bands were observed on X-ray film by using an enhanced chemiluminescence system (Pierce Biotechnology). To determine the linear range of the chemiluminescence signals, the X-ray film was quantitatively analyzed via densitometry.

Statistical analysis. All experiments were repeated three times and similar results were obtained. Statistical analysis was performed using the SPSS 11.5 statistics software (SPSS, Inc., Chicago, IL). Data are expressed as the means \pm SEM with the number of individual experiments described in the figure legends. P-values $<0.05$ were considered statistically significant.

\section{Results}

Cytotoxic effects of etoposide in the treatment of HepG2 cells. The cytotoxic effect of etoposide treatment on HepG2 cells was examined with an MTT assay. The cell viability was inhibited by etoposide $(0-80 \mu \mathrm{g} / \mathrm{ml})$ in a dose- and time-dependent manner. The cytotoxicity rate increased with etoposide treatment at the concentration of $20 \mu \mathrm{g} / \mathrm{ml}$ for $24 \mathrm{~h}$, and reached $49.7 \%$ at $48 \mathrm{~h}$, and $63.8 \%$ at $72 \mathrm{~h}$ (Fig. 1A). The $\mathrm{IC}_{50}$ for $48 \mathrm{~h}$ with etoposide treatment in HepG2 cells was $20 \pm 0.92 \mu \mathrm{g} / \mathrm{ml}$. Based on these results, $20 \mu \mathrm{g} / \mathrm{ml}$ of etoposide in HepG2 cells for $48 \mathrm{~h}$ were used in further experiments.

Autophagy induced by etoposide. To test whether autophagy was stimulated after exposure to etoposide in HepG2 cells, three different methods, GFP-LC3 modulation by fluorescent microscopy, LC3 conversion on a Western blot and electron microscopy, were used. LC3 is a mammalian homologue of Atg8, a protein that is essential for autophagy in yeast and that is recruited to the autophagosome membrane in an Atg5-dependent manner (16). GFP-LC3, LC3 fused to green fluorescent protein, has been usually employed to monitor autophagy, measured as an elevation in punctate GFP-LC3 detected by fluorescence microscopy. As shown in Fig. 1B, punctate GFP-LC3 structures accumulated in the cytoplasm of etoposide-treated cells, but remained at low levels in the untreated cells (control). Immunoblotting analysis was used to examine the expression of LC3 protein. Immunoblotting of LC3 usually exhibits two bands: LC3-I and LC3-II. Given 
A

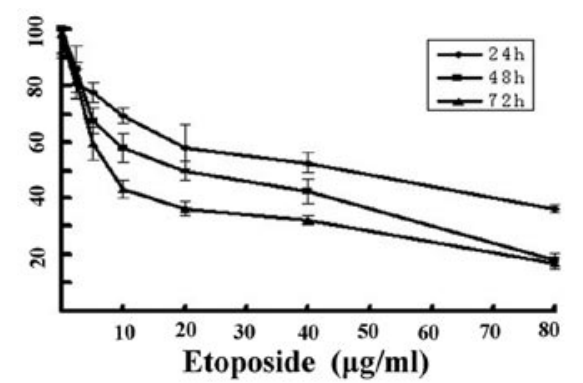

B
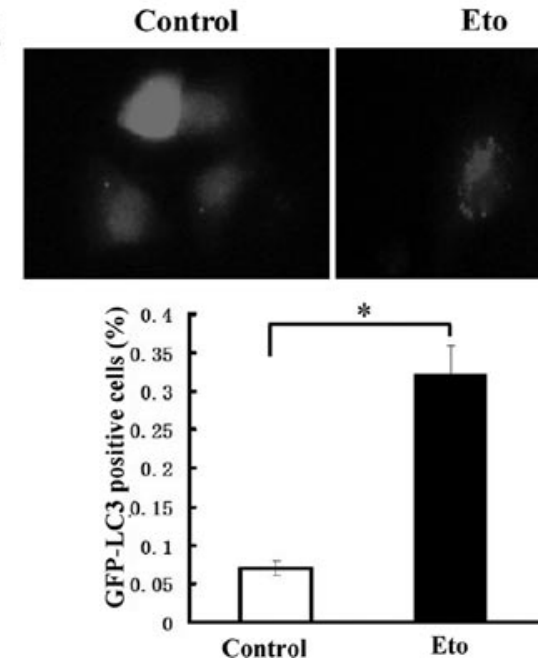

C

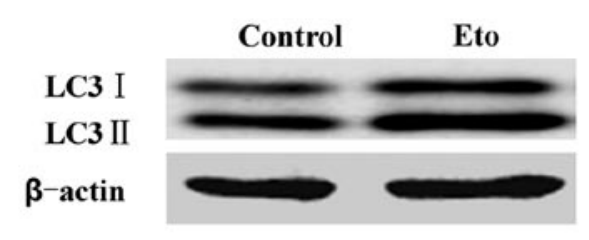

D
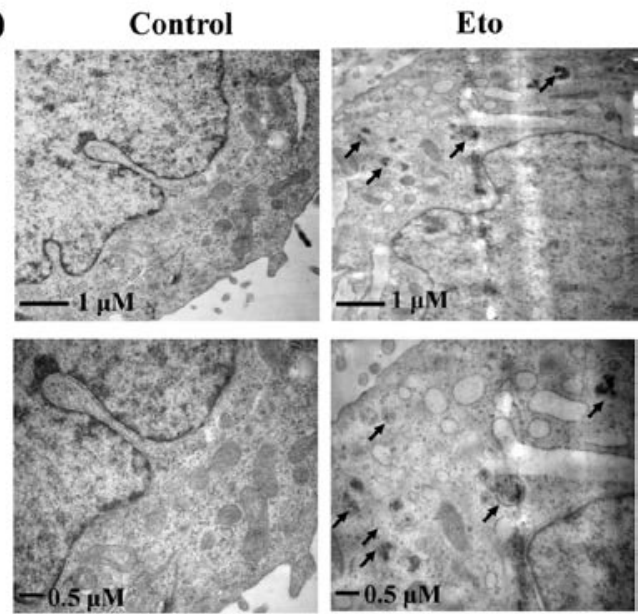

Figure 1. Etoposide inhibits proliferation and induces autophagy in HepG2 cells. (A) The effects of etoposide (0-80 $\mu \mathrm{g} / \mathrm{ml})$ on the regression of HepG2 cells for $24 \mathrm{~h}, 48 \mathrm{~h}$ or $72 \mathrm{~h}$ were examined by the MTT assay. (B) Cells were treated with $20 \mu \mathrm{g} / \mathrm{ml}$ etoposide (Eto) for $48 \mathrm{~h}$, and GFP-LC3 was viewed with a fluorescence microscope. Representative images of GFP-LC3 fluorescence are shown. *p<0.05, significant difference of GFP-LC3 positive cells (\%) in etoposide-treated cells compared to untreated cells (control). (C) Western blot analysis of the expression of LC3 in etoposide-treated cells as compared with control; $\beta$-actin was employed as a protein loading control. (D) Representative electron microscopy images were obtained from etoposide-treated cells and compared to control. The typical autophagosomes or autolysosomes are denoted by the arrows.

that LC3-II is much more sensitive than LC3-I, the amount of LC3-II is more appropriate for detecting autophagy in mammalian cells. Indeed, up-regulation of LC3-II was detected in etoposide-treated cells (Fig. 1C). Finally, transmission electron microscopy (TEM), one of the most reliable methods for autophagy (17), was used to further confirm the cumulative autophagic activities in etoposide-treated cells. The data of TEM showed that the autophagosomes (a double membrane structure) and autolysosomes (a single membrane structure) were readily observed in etoposide-treated cells, but were rarely observed in untreated cells (Fig. 1D). Taken together, these findings indicate that autophagy was stimulated in HepG2 cells after exposure to etoposide.

Autophagy inhibition by 3-methyladenine enhances etoposide-induced cell death. Autophagy has a dual role of either programmed cell death or cytoprotection. To investigate the function of etoposide-induced autophagy, 3MA, a general inhibitor of autophagy (18), was used to suppress autophagy in etoposide-treated cells. Initially, etoposide treatment caused the increase of GFP-LC3 dots, which was overcome by addition of 3MA. In addition, a large amount of GFP-LC3 dots were observed in HepG2 cells after exposure to rapamycin, a known inducer of autophagy (19) (Fig. 2A). Similarly, immunoblotting analysis confirmed that down-regulation of LC3-II was detected in etoposide-treated cells co-treated with 3MA (Fig. 2B). We used the Annexin V-FITC/PI assay to detect the changes of cell death. Using this assay, viable cells (Annexin $\mathrm{V}^{-} / \mathrm{PI}^{-}$), early apoptotic cells (Annexin $\mathrm{V}^{+} / \mathrm{PI}^{-}$), late apoptotic or necrotic cells (Annexin $\mathrm{V}^{+} / \mathrm{PI}^{+}$) are easily classified (20). As shown in Fig. 2C, there was a significant difference in cell death between the combined group (etoposide+3MA) and the etoposide group $(\mathrm{p}<0.05)$. Altogether, these results illustrate that inhibition of autophagy by 3MA enhanced etoposide-induced cell death in HepG2 cells.

Blockage of autophagy by beclin-1 siRNA enhances etoposide-induced cell death. We examined the expression of beclin-1, another molecule that is known to be required for the formation of autophagosomes (21). There was no change in the levels of beclin-1 protein between etoposide treatment and control, but the expression of beclin-1 was down-regulated with the addition of 3MA and up-regulated with rapamycin (Fig. 3A). Then, specific siRNA targeted against beclin-1 was used to better understand cell responses caused by inhibition of autophagy and its function in the increased autophagy after exposure to etoposide. The specific siRNA duplex resulted in at least $90 \%$ decline of beclin-1 protein as compared with control siRNA (Fig. 3B). As presented in Fig. 3B and C, autophagy was suppressed by beclin-1 siRNA, measured as the down-regulation of LC3-II and the decline of GFP-LC3 positive cells. Subsequently, we explored whether suppression 
A
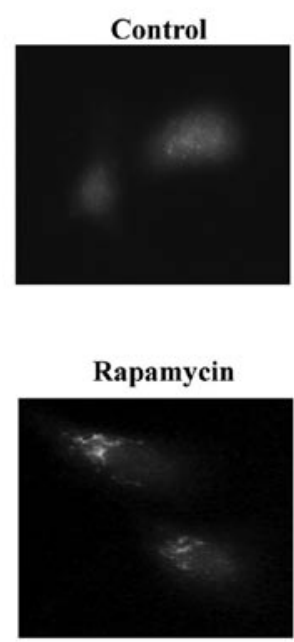

3 MA

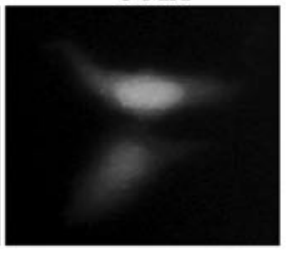

Eto

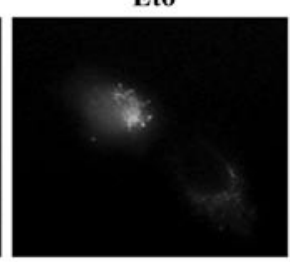

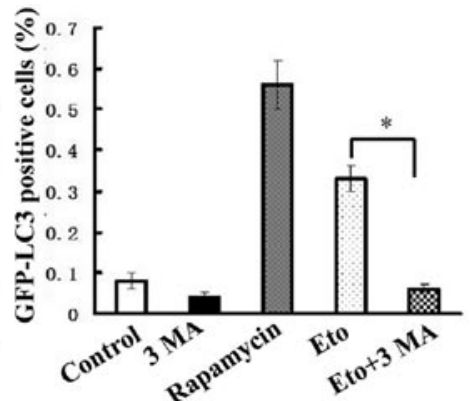

Eto+3 MA

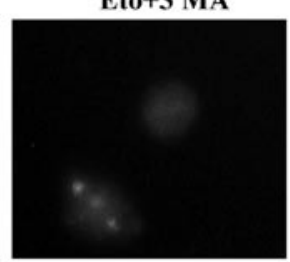

B

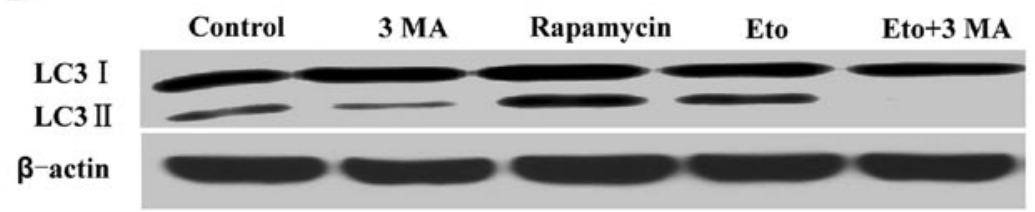

C

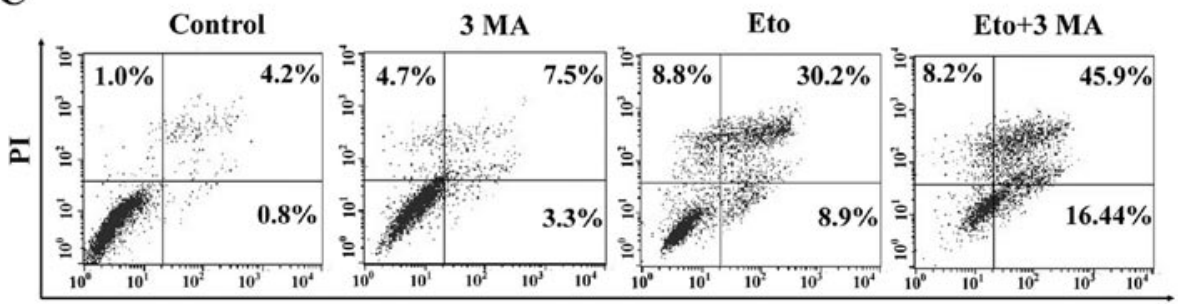

ANNEXIN-V

Figure 2. Autophagy inhibition by 3MA enhances etoposide-induced cell death. (A) HepG2 cells were divided into five groups: untreated (control), $2.5 \mathrm{mM}$ of 3MA (3MA), $20 \mu \mathrm{g} / \mathrm{ml}$ of etoposide (Eto), $20 \mu \mathrm{g} / \mathrm{ml}$ of etoposide in combination with $2.5 \mathrm{mM}$ of 3MA (Eto+3MA), and $10 \mathrm{nM}$ rapamycin (rapamycin). Representative images of GFP-LC3 by fluorescence microscopy on cells of the five groups were obtained. GFP-LC3 positive cells (\%) were presented as the mean \pm SEM from the three independent experiments. "p $<0.05$ denotes a significant difference between Eto and Eto +3 MA. (B) Cell lysates of the five groups were prepared and subjected to immunoblotting with antibodies to LC3 and $\beta$-actin. (C) Annexin V/PI analysis detected apoptosis of cells between the combination group (Eto+3MA) and Eto. The percentage of Annexin V and/or propidium iodide (PI) positive cells are depicted with typical cytofluorometer quadrant graphs. The apoptotic index was $39.1 \%$ for Eto, $62.3 \%$ for Eto+3MA.

of autophagy by RNAi enhanced etoposide-induced cell death or not. After etoposide treatment, beclin-1-siRNA transfectants demonstrted a $32.7 \%$ increase of apoptotic and necrotic cell death in comparison with control-siRNA transfectants (Fig. 3D). These findings show that autophagy inhibition by beclin-1 siRNA also strengthened DNA damaged-induced cell death and suggested that beclin-1 was important for the induction of autophagy in HepG2 cells under etoposide treatment.

DNA damage-induced autophagy is not dependent on p53 activation. It has been reported that genotoxic stress and DNA damage can promote autophagy through a p53-dependent mechanism (22). Increased expression of the p53 protein level was detected in etoposide-treated cells in accordance to previous reports (Fig. 4A). These facts prompted us to test whether etoposide-induced autophagy was linked to p53 activation. For this purpose, PFTa, a pharmacological antagonist of p53, was used to co-treat etoposide-treated cells. As shown in Fig. 4B and C, GFP-LC3 dots and the amount of LC3-II increased significantly and to a greater extent with PFTa co-treatment than with etoposide alone. Next, the Annexin V/ PI assay demonstrated that the proportion of cell death was $44.3 \%$ in etoposide-treated cells, and slightly decreased in the combination group to $31.5 \%$ (Fig. 4D). These results suggest that etoposide-induced autophagy is not dependent on p53 activation.

AMPK is involved in etoposide-induced autophagy. Since p53 protein presented an indirect effect to DNA damageinduced autophagy, we first analyzed the status of the mTOR signaling pathway in HepG2 cells after exposure to etoposide. Phosphorylation of 70s6K at Thr389, which has been widely described to reflect mTOR activity (23), was reduced prominently in etoposide-treated as compared with the untreated cells (Fig. 5A). In combination with our previous results (Fig. 2A and C), we have demonstrated that the elevation of autophagy activity observed in etoposide-treated cells was linked to mTOR pathway inhibition. 
A

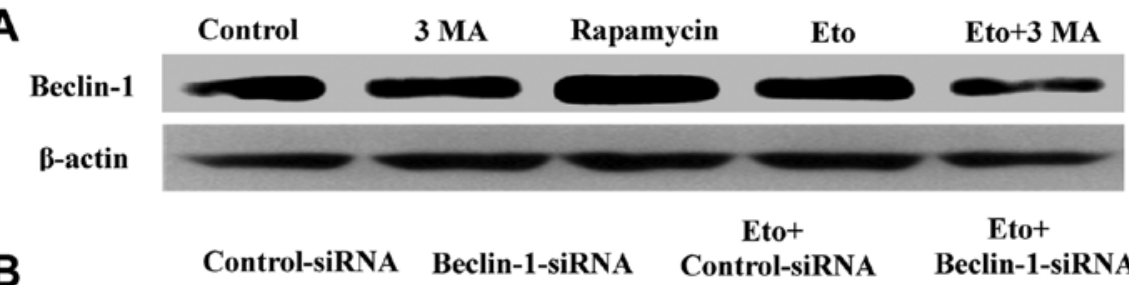

B

Beclin-1

LC3 I

LC3 II

B-actin

Control-siRNA Beclin-1-siRNA Control-siRNA Beclin-1-siRNA

C

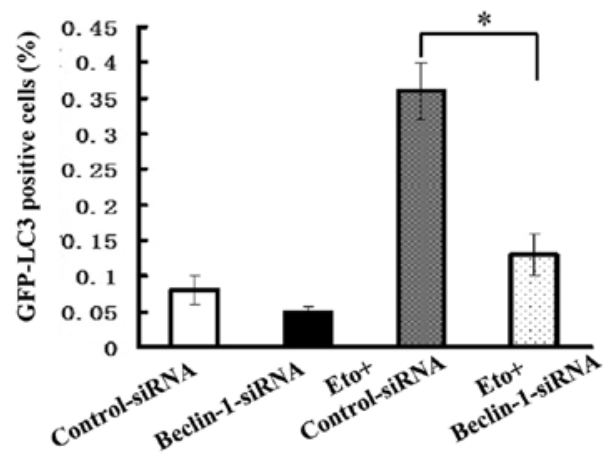

D

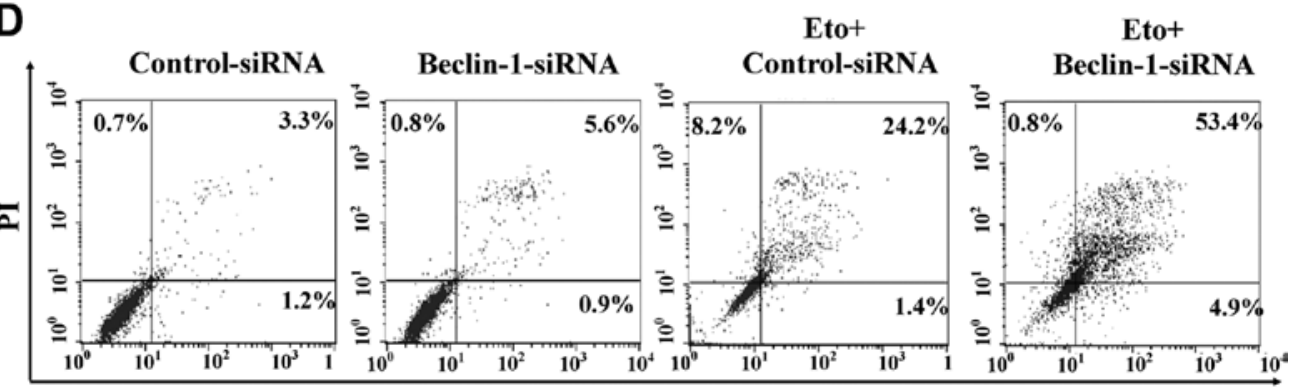

ANNEXIN- $\mathrm{V}$

Figure 3. Autophagy inhibition enhances etoposide-induced cell death. (A) Cell lysates of the five groups (indicated in Fig. 2) were prepared and subjected to immunoblotting with antibodies to beclin-1 and $\beta$-actin. (B) Beclin-1 was knocked down by RNAi interference in HepG2 cells, and led to the decline of LC3-II after etoposide (Eto) treatment. (C) After exposure to Eto, beclin-1-siRNA transfected cells (Beclin-1 siRNA+Eto) was modulated by GFP-LC3 localization as compared with control-siRNA transfected cells (Control siRNA+Eto). Significant difference of GFP-LC3 positive cells (\%) between beclin-1siRNA transfected cells and control-siRNA transfected cells are presented ( $" \mathrm{p}<0.05)$. (D) Apoptosis of targeting beclin-1 after exposure to Eto was detected by Annexin V/PI analysis. The percentage of Annexin V and/or propidium iodide (PI) positive cells, Control siRNA+Eto (25.6\%) and Beclin-1 siRNA+Eto $(58.3 \%)$ respectively, is shown.

Given that mTOR is negatively regulated by AMPK, we next analyzed the AMPK activity to test whether a possible alteration in this kinase could account for the increased autophagic process in etoposide-treated cells. For this purpose, we first examined the phosphorylation status of AMPKa at Thr172, which has been widely used to monitor AMPK activity (24). AMPK phosphorylation was significantly higher in etoposide-treated than in untreated cells (Fig. 5A). These results suggested that etoposide activated AMPK and accordingly inhibited mTOR, accompanied with induction of autophagy in HepG2 cells.

Suppression of AMPK enhances etoposide-induced cell death. To investigate the role of AMPK in etoposide-induced autophagy, blockage of AMPK using RNAi was employed in etoposide-treated cells. Western blot analysis firstly indicated that the expression of AMPK $\alpha$ was suppressed at least $80 \%$ by the specific siRNA duplex as compared with the control siRNA (Fig. 5B). Furthermore, the increase of LC3-II and GFP-LC3 dots after etoposide treatment was inhibited in AMPK $\alpha$-siRNA transfectants, in contrast to control-siRNA transfectants, showing a down-regulation of the autophagic activities by AMPK siRNA (Fig. 5C and D). Subsequently, we explored whether inhibition of AMPK enhanced etoposideinduced cell death. As presented in Fig. 6A, after exposure to etoposide, there was a nearly double elevation of apoptotic and necrotic cells between AMPK $\alpha$-siRNA transfectants and control-siRNA transfectants. Moreover, similar results were obtained by flow cytometry when etoposide was combined with compound $\mathrm{C}$, a specific inhibitor of AMPK (Fig. 6B) (25). These results demonstrate that suppression of AMPK activity enhanced etoposide-induced cell death. 

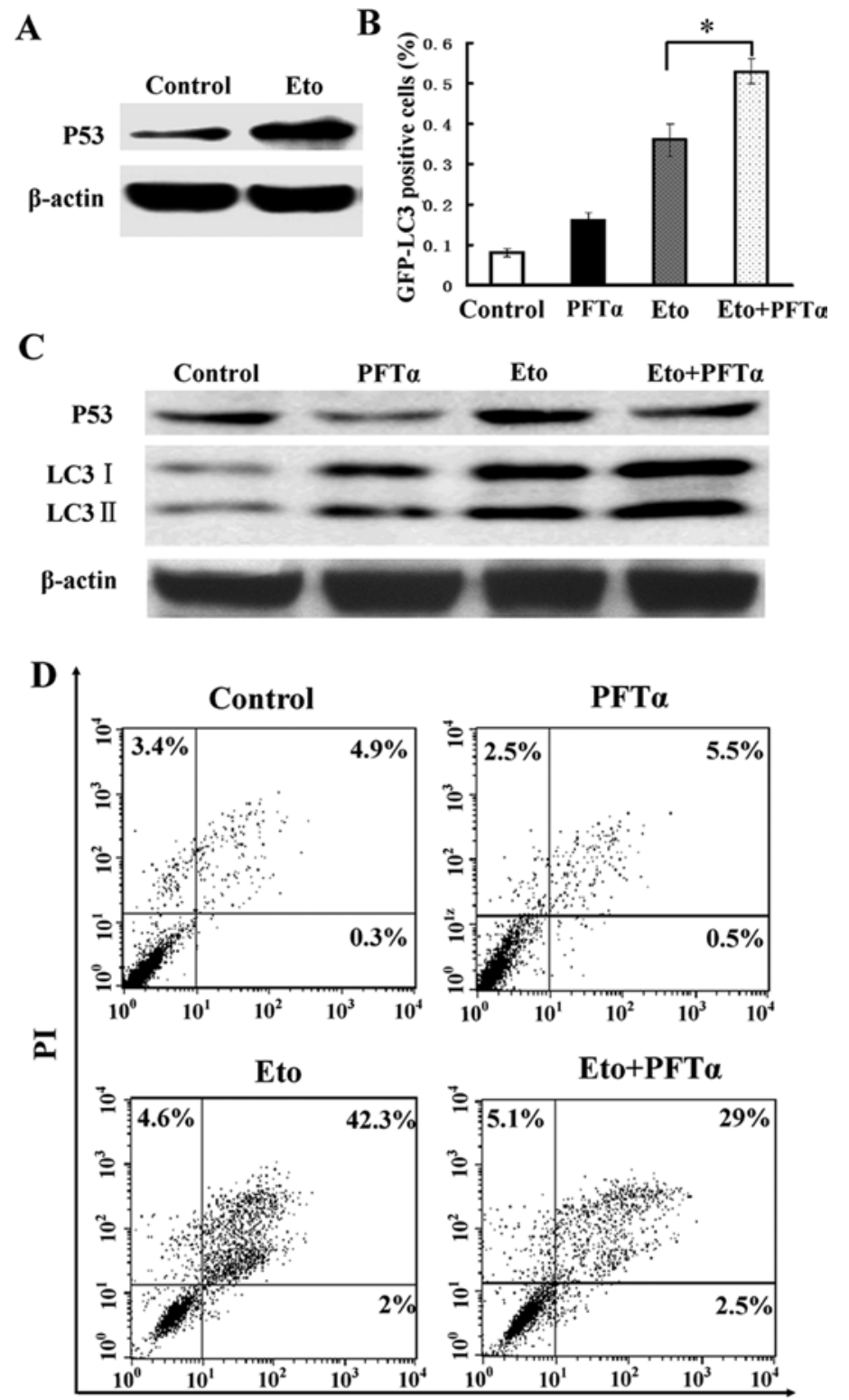

ANNEXIN-V

Figure 4. Etoposide-induced autophagy is not dependent on p53 activation. (A) Western blot analysis demonstrated that p53 is activated in etoposide-treated cells. (B) After etoposide treatment, p53 inhibition using PFT $\alpha$ (Eto+PFT $\alpha$ ) caused the augmentation of GFP-LC3 positive cells, compared to etoposide-treated cells ( $\mathrm{p}<0.05)$. (C) p53 inhibition using PFTa led to the elevation of LC3-II after etoposide treatment. (D) Flow cytometry assay of cell death in cells treated with etoposide or etoposide combined with PFT $\alpha$ (Eto+PFT $\alpha$ ). The percentage of Annexin V and/or PI positive cells, Eto (44.3\%) and Eto+PFT $\alpha(31.5 \%)$ is shown.

\section{Discussion}

The present study demonstrated that etoposide induces autophagy in HepG2 cells. Autophagy has been frequently detected by antineoplastic agents in a great number of cancer cells, such as cervical carcinoma cells (26), human glioma cells (27) or human colon cancer cells (28). Inhibition of autophagy by 3MA enhanced etoposide-induced cell death in HepG2 cells. The autophagic process is protective against etoposide treatment. Stimulation of autophagy might function to sequester and degrade proteins or organelles such as mitochondria that are damaged by etoposide treatment to release amino acid and maintain hepatic cancer cell viability.

Our findings have shown that depletion of beclin- 1 causes a significant elevation of etoposide-induced cell death. Beclin-1, the mammalian analogue of Atg6, has been known to stimulate the formation of autophagy (29). At the early stage of tumor development, beclin-1 functions as a tumor suppressor, but promotes tumor growth at the later stages of tumor progression (30). Our studies have demonstrated that beclin-1 protein was required for inhibition of apoptosis and DNA damage by etoposide in HepG2 cells. The down-regulation of beclin-1 by 3MA switched autophagy to apoptosis in HCC following etoposide and rapamycin treatment, leading to the up-regulation of beclin-1 and to stimulation of autophagy followed by the absence of apoptosis in HepG2 cells. We have also demonstrated that the autophagic survival response to DNA damage was undone when beclin-1 was blocked by siRNA, and this resulted in an increase of hepatic cancer cell death. Similarly, Abedin et al have reported that down- 
A

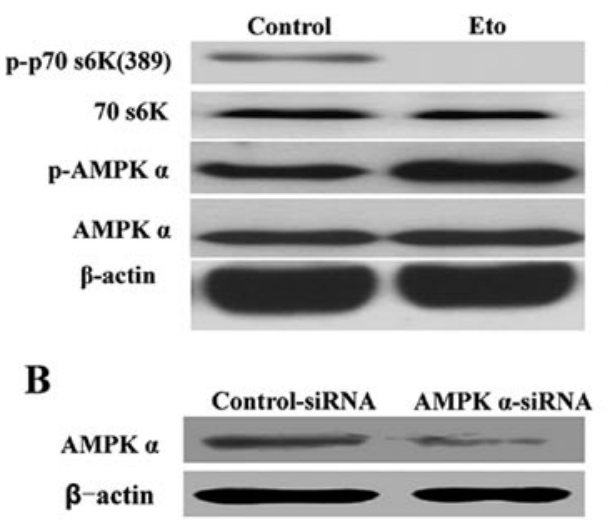

C

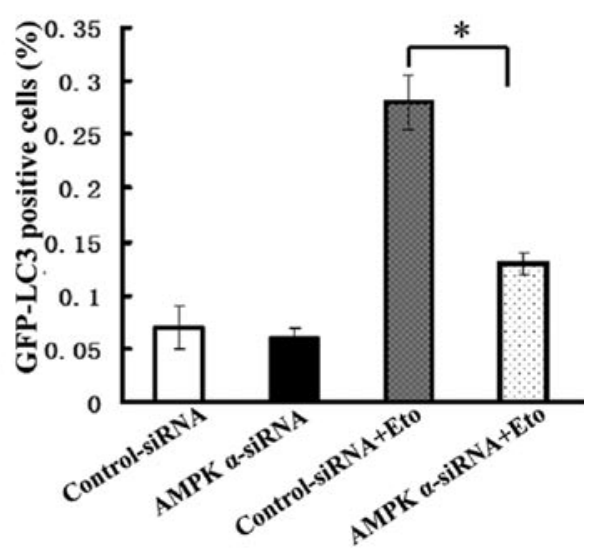

D

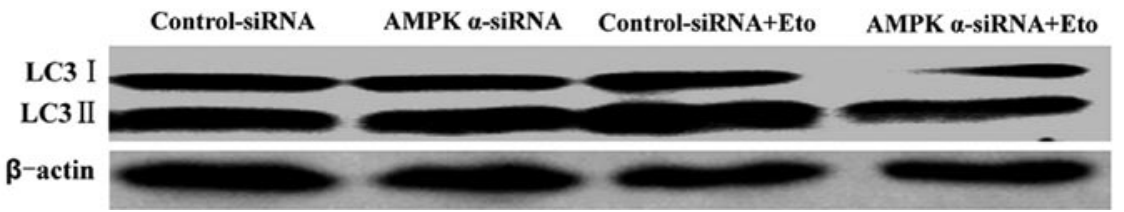

Figure 5. Etoposide-stimulated autophagy is involved in activation of AMPK (A) Immunoblotting analysis of the expression of p-AMPKa (Thr172) or p-p70s6K (Thr389) in etoposide-treated cells as compared with control. (B) AMPK was knocked down successfully by RNAi in HepG2 cells. (C) After etoposide treatment, AMPK blockage caused the reduction of GFP-LC3 positive cells. ${ }^{*} p<0.05$, significant difference between AMPK $\alpha$-siRNA transfected cells and control-siRNA transfected cells. (D) AMPK knockdown led to the decline of LC3-II after etoposide treatment.
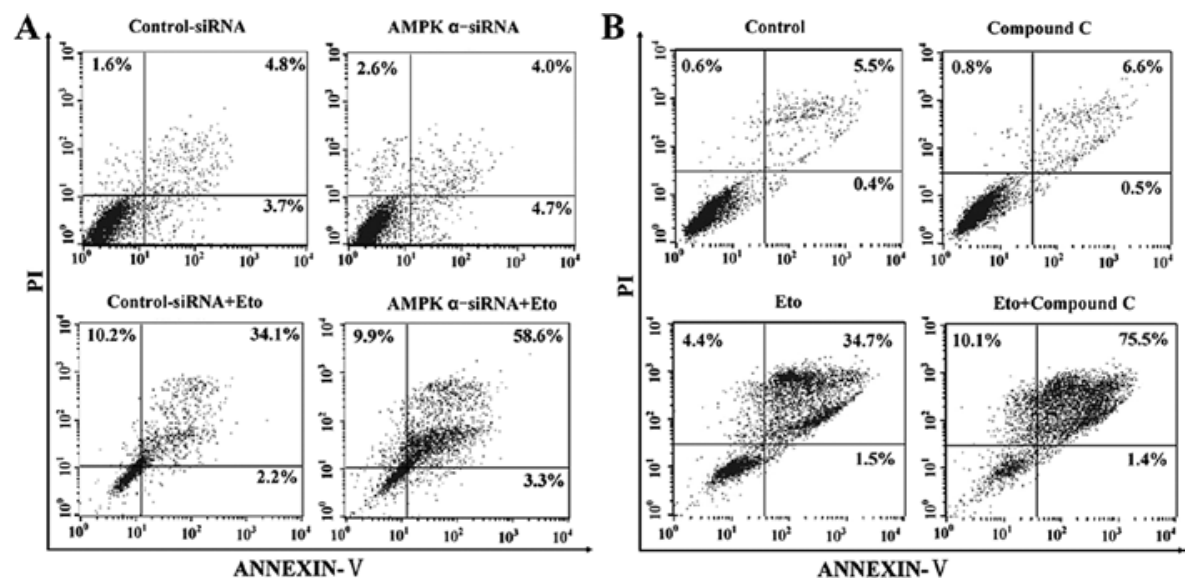

Figure 6. Inhibition of AMPK enhances etoposide-induced cell death. Annexin V/PI analysis showed a significant elevation of cell death small after inhibition of AMPK using interfering RNA (A) and compound C (B) in etoposide-treated cells.

regulation of beclin-1 led to cell death in response to DNA damage in MCF cells (31). In HCC cells, basal high expression of beclin-1 protein has a defensive mechanism possibly as a result of prevention of the initiation of apoptosis in hepatic cancer cells under etoposide treatment.

We provide further evidence that the autophagic activities are not dependent on p53 activation, yet are strongly related to AMPK activity. Inhibition of p53 by PFT $\alpha$ promoted cell adaptation through enhanced autophagy during etoposide treatment of HepG2 cells. PFT $\alpha$ treatment has also been reported to protect against antineoplastic agent-induced apoptosis $(32,33)$. Moreover, recent data have demonstrated that inactivation of p53 induces accumulation of autophagosomes, and depletion of cytoplasmic, not nuclear p53, contributing to stimulation of autophagy (34). Our results indicate that stimulation of autophagy by etoposide is regulated by mTOR inhibition, associated with AMPK activation. There is growing evidence supporting that AMPK, a major mediator of energy balance, plays an essential role in increased autophagic activities through mTOR inhibition under stressful conditions (35). In response to DNA damage, activation of AMPK through inhibition of mTOR stimulates autophagy in MEF (22). Papandreou et al (36) demonstrated that AMPK is involved in hypoxia-induced autophagy. However, AMPK activation by AICA riboside (AICAR), suppresses the autophagic activities in hepatocytes (37). The role of the AMPK signaling 
pathway in autophagy is not well understood. Our studies have shown that AMPK inhibition leads to a decline of etoposide-induced autophagic activities. Inactivation of AMPK converted a cell survival response into a cell death process in response to DNA damage. Similarly, AMPK downregulation increases cisplatin-induced tumor cell death (27). Inhibition of autophagy through siRNA-targeted AMPK may abrogate the expression of anti-apoptotic proteins and thereby initiate the apoptosis pathway.

In summary, etoposide-induced autophagy is linked to AMPK activation. Manipulation of autophagy for therapy may provide a useful method to overcome chemoresistance. Therefore, AMPK inhibitors are promising drug candidates for treating hepatocellular carcinoma.

\section{Acknowledgements}

This study was supported by the National Natural Science Foundation of China (Grant no.: 30772859). The authors thank Dr Meng Qiang Li for his professional support and help.

\section{References}

1. Keehn DM and Frank-Stromborg M: A worldwide perspective on the epidemiology and primary prevention of liver cancer. Cancer Nurs 14: 163-174, 1991.

2. Tanaka H, Tsukuma $\mathrm{H}$ and Oshima A: Epidemiology of primary liver cancer in Japan. Gan To Kagaku Ryoho 28: 151-154, 2001 (In Japanese).

3. Bosch FX, Ribes J and Borras J: Epidemiology of primary liver cancer. Semin Liver Dis 19: 271-285, 1999.

4. Yasuda H, Fujino H, Tagawa K and Unuma T: Hepatic arterial infusion of etoposide in the treatment of primary liver neoplasms. Gan To Kagaku Ryoho 17: 381-384, 1990 (In Japanese).

5. Kinebuchi Y, Noguchi W, Igawa Y and Nishizawa O: Recurrent retroperitoneal malignant nerve sheath tumor associated with neurofibromatosis type 1 responding to carboplatin and etoposide combined chemotherapy. Int J Clin Oncol 10: 353-356, 2005.

6. Wong LC, Collins RJ, Ngan HY and Ma HK: Etoposide combination chemotherapy in refractory ovarian malignant germ cell tumor. Gynecol Oncol 39: 123-126, 1990.

7. Baldwin EL and Osheroff N: Etoposide, topoisomerase II and cancer. Curr Med Chem Anticancer Agents 5: 363-372, 2005.

8. Yin D, Tamaki $\mathrm{N}$ and Kokunai T: Wild-type p53-dependent etoposide-induced apoptosis mediated by caspase-3 activation in human glioma cells. J Neurosurg 93: 289-297, 2000.

9. Cavalli F, Rozencweig M, Renard J, Goldhirsch A and Hansen HH: Phase II study of oral VP-16-213 in hepatocellular carcinoma. Eur J Cancer Clin Oncol 17: 1079-1082, 1981.

10. Falkson G, MacIntyre JM, Moertel CG, Johnson LA and Scherman RC: Primary liver cancer. An Eastern Cooperative Oncology Group Trial. Cancer 54: 970-977, 1984.

11. Mizushima N: Autophagy: process and function. Genes Dev 21: 2861-2873, 2007.

12. Li M, Jiang X, Liu D, Na Y, Gao GF and Xi Z: Autophagy protects LNCaP cells under androgen deprivation conditions Autophagy 4: 54-60, 2008.

13. Shackelford DB and Shaw RJ: The LKB1-AMPK pathway: metabolism and growth control in tumour suppression. Nat Rev Cancer 9: 563-575, 2009.

14. Levine YC, Li GK and Michel T: Agonist-modulated regulation of AMP-activated protein kinase (AMPK) in endothelial cells. Evidence for an AMPK $\rightarrow$ Rac1 $\rightarrow \mathrm{Akt} \rightarrow$ endothelial nitric-oxide synthase pathway. J Biol Chem 282: 20351-20364, 2007.

15. Meley D, Bauvy C, Houben-Weerts JH, et al: AMP-activated protein kinase and the regulation of autophagic proteolysis. J Biol Chem 281: 34870-34879, 2006.
16. Kabeya Y, Mizushima N, Ueno T, et al: LC3, a mammalian homologue of yeast Apg8p, is localized in autophagosome membranes after processing. EMBO J 19: 5720-5728, 2000.

17. Kabeya Y, Mizushima N, Yamamoto A, Oshitani-Okamoto S, Ohsumi Y and Yoshimori T: LC3, GABARAP and GATE16 localize to autophagosomal membrane depending on form-II formation. J Cell Sci 117: 2805-2812, 2004.

18. Seglen PO and Gordon PB: 3-Methyladenine: specific inhibitor of autophagic/lysosomal protein degradation in isolated rat hepatocytes. Proc Natl Acad Sci USA 79: 1889-1892, 1982.

19. Codogno P and Meijer AJ: Autophagy and signaling: their role in cell survival and cell death. Cell Death Differ 12 (Suppl. 2): 1509-1518, 2005.

20. Wang L, Yu C, Lu Y, et al: TMEM166, a novel transmembrane protein, regulates cell autophagy and apoptosis. Apoptosis 12: 1489-1502, 2007.

21. Liang XH, Jackson S, Seaman M, et al: Induction of autophagy and inhibition of tumorigenesis by beclin 1 . Nature 402: 672-676, 1999.

22. Feng Z, Zhang H, Levine AJ and Jin S: The coordinate regulation of the p53 and mTOR pathways in cells. Proc Natl Acad Sci USA 102: 8204-8209, 2005.

23. Pullen $\mathrm{N}$ and Thomas G: The modular phosphorylation and activation of p70s6k. FEBS Lett 410: 78-82, 1997.

24. Shaw RJ, Kosmatka M, Bardeesy N, et al: The tumor suppressor LKB1 kinase directly activates AMP-activated kinase and regulates apoptosis in response to energy stress. Proc Natl Acad Sci USA 101: 3329-3335, 2004.

25. Zhou G, Myers R, Li Y, et al: Role of AMP-activated protein kinase in mechanism of metformin action. J Clin Invest 108: 1167-1174, 2001.

26. Lee SB, Tong SY, Kim JJ, Um SJ and Park JS: Caspaseindependent autophagic cytotoxicity in etoposide-treated CaSki cervical carcinoma cells. DNA Cell Biol 26: 713-720, 2007.

27. Harhaji-Trajkovic L, Vilimanovich U, Kravic-Stevovic T, Bumbasirevic V and Trajkovic V: AMPK-mediated autophagy inhibits apoptosis in cisplatin-treated tumor cells. J Cell Mol Med 13: 3644-3654, 2009.

28. Nishikawa T, Tsuno NH, Okaji Y, et al: Inhibition of autophagy potentiates sulforaphane-induced apoptosis in human colon cancer cells. Ann Surg Oncol 17: 592-602, 2010.

29. Pattingre S, Tassa A, Qu X, et al: Bcl-2 antiapoptotic proteins inhibit Beclin 1-dependent autophagy. Cell 122: 927-939, 2005.

30. Kondo Y, Kanzawa T, Sawaya R and Kondo S: The role of autophagy in cancer development and response to therapy. Nat Rev Cancer 5: 726-734, 2005.

31. Abedin MJ, Wang D, McDonnell MA, Lehmann U and Kelekar A: Autophagy delays apoptotic death in breast cancer cells following DNA damage. Cell Death Differ 14: 500-510, 2007.

32. Shi YK, He XH, Han XH, et al: Mobilization of autologous peripheral blood stem cells with etoposide and recombinant human granulocyte colony stimulating factor in malignant tumor patients. Zhonghua Zhong Liu Za Zhi 26: 360-363, 2004 (In Chinese).

33. Zhang M, Liu W, Ding D and Salvi R: Pifithrin-alpha suppresses p53 and protects cochlear and vestibular hair cells from cisplatininduced apoptosis. Neuroscience 120: 191-205, 2003.

34. Tasdemir E, Maiuri MC, Galluzzi L, et al: Regulation of autophagy by cytoplasmic p53. Nat Cell Biol 10: 676-687, 2008.

35. Matsui Y, Takagi H, Qu X, et al: Distinct roles of autophagy in the heart during ischemia and reperfusion: roles of AMP-activated protein kinase and Beclin 1 in mediating autophagy. Circ Res 100: 914-922, 2007.

36. Papandreou I, Lim AL, Laderoute K and Denko NC: Hypoxia signals autophagy in tumor cells via AMPK activity, independent of HIF-1, BNIP3, and BNIP3L. Cell Death Differ 15: 1572-1581, 2008.

37. Samari HR and Seglen PO: Inhibition of hepatocytic autophagy by adenosine, aminoimidazole-4-carboxamide riboside, and N6-mercaptopurine riboside. Evidence for involvement of Amp-activated protein kinase. J Biol Chem 273: 23758-23763, 1998. 